\section{BMJ Open} Ophthalmology

\title{
Ophthalmic phenotype of TCIRG1 gene mutations in Chinese infantile malignant osteopetrosis
}

\author{
Wenhong Cao, ${ }^{1,2}$ Wenbin Wei, ${ }^{2}$ Qian $\mathrm{Wu}^{1}$
}

To cite: Cao W, Wei W, Wu Q. Ophthalmic phenotype of TCIRG1 gene mutations in Chinese infantile malignant osteopetrosis. BMJ Open Ophthalmology 2018;3:e000180. doi:10.1136/ bmjophth-2018-000180

Received 30 May 2018 Revised 25 September 2018 Accepted 26 September 2018
Check for updates

C Author(s) (or their employer(s)) 2018. Re-use permitted under CC BY-NC. No commercial re-use. See rights and permissions. Published by BMJ.

${ }^{1}$ Department of Ophthalmology, Beijing Children's Hospital, National Center for Children's Health, National Key Discipline of Pediatrics, Capital Medical University, Beijing, China ${ }^{2}$ Beijing Tongren Eye Center, Beijing Key Laboratory of Ophthalmology and Visual Science, Beijing Key Laboratory of Intraocular Tumor Diagnosis and Treatment, Beijing Tongren Hospital, Capital Medical University, Beijing, China

Correspondence to DrWenbin Wei; weiwenbintr@ 163.com

\section{ABSTRACT}

Objective To evaluate the ophthalmic phenotypes associated with T-cell immune regulator 1 (TCIRG1) mutations in Chinese patients with infantile malignant osteopetrosis (IMO).

Methods and analysis 27 Chinese TCIRG1-related osteoporosis infants were enrolled using direct DNA sequencing of PCR-amplified exons. 12 cases had frameshift mutation (the frameshift mutation group, group F), and 15 cases had point mutation (the point mutation group, group P). The clinical features of the two groups were compared, including age at onset, gaze qualities, optic atrophy, optic canal stenosis and waveforms of Flash visual-evoked potential (FVEP).

Results The clinical signs, except age at onset and FVEP, showed statistically significant differences between the two groups. The mean age at onset was 1.8 months in group $\mathrm{F}$ and 4.3 months in group P; 22 eyes (92\%) with frameshift mutation and $16(53 \%)$ with point mutation had poor gaze qualities, such as nystagmus and/or strabismus; optic atrophy was found in 16 eyes (67\%) in group $F$ and $6(20 \%)$ in group P; the average optic canal diameter was $1.45 \mathrm{~mm}$ in the frameshift mutation cases, $1.87 \mathrm{~mm}$ in other cases; FVEP indicated that the waveforms in 10 eyes (42\%) were not elicited in group $F$, yet five eyes $(17 \%)$ in group P.

Conclusion In Chinese TCIRG1-related patients of IMO, the optic canal stenosis and optic atrophy were more serious in cases with frameshift mutations. However, no differences in the conduction block of optic nerve were found between the two groups.

\section{INTRODUCTION}

To date, more than 10 osteopetrosis-causing genes have been identified. ${ }^{1}$ The inheritance patterns include autosomal dominant, autosomal intermediate, autosomal recessive and $\mathrm{X}$ linked. The disease severity also varies from malignant, intermediate, to mild manifestations. Infantile malignant osteopetrosis (IMO) is an extremely rare congenital disease caused by bone marrow failure resulting from osteoclast dysfunction. This disorder is characterised by neonatal and infantile onset, a systemic sclerosis of bones, vulnerability to fracture, progressive anaemia, infection, hepatosplenomegaly and cranial nerve dysfunction. The rapid progression

\section{Key messages}

What is already known about this subject?

- T-cell immune regulator 1 (TCIRG1) is one of the main genes that are responsible for the majority of infantile malignant osteopetrosis (IMO) cases, which are characterised by neonatal and infantile onset, a systemic sclerosis of bones, vulnerability to fracture, progressive anaemia, infection, hepatosplenomegaly and cranial nerve dysfunction, including poor gaze qualities, optic atrophy and optic canal stenosis.

To our knowledge, there is no study on the ophthalmic phenotypes of TCIRG1 mutations in patients with IMO.

\section{What are the new findings?}

We found that by comparing frameshift and point mutation cases in 27 Chinese patients with TCIRG1IMO, severity of the ocular manifestations was related to the type of gene mutation, and the frameshift mutation in TCIRG1 led to more serious phenotypes.

How might these results change the focus of research or clinical practice?

Future studies should assess the correlation of TCIRG1 gene mutation and the mechanism of ocular sign occurrence.

- This may encourage better understanding of the mechanism of ocular sign occurrence and more targeted treatment of IMO

and severity contribute to a high mortality. ${ }^{2}$ It is inherited as an autosomal recessive trait, with an incidence of 1 in 250000 births in North America, particularly high incidence reported in Costa Rica (3.4:100 000). ${ }^{3}$

Currently, T-cell immune regulator 1 (TCIRG1, Gene ID: 10312, MIM 604592) ${ }^{4}$ is one of the main genes that are responsible for more than $50 \%$ of IMO cases. ${ }^{56}$ It has been reported that homozygous mutations in the genes encoding the a3 subunit of VATPase (TCIRG1) produce severe malignant osteopetrosis phenotypes in both humans and mice. ${ }^{4}$ Mutations in TCIRG1 associated with general clinical features have been reported in previous studies. ${ }^{78}$ In this study, we present a more detailed investigation of the relation 
between the ophthalmic phenotype of Chinese patients with IMO and the genotype of TCIRG1 mutation.

\section{SUBJECTS AND METHODS}

The study was conducted in accordance with the Declaration of Helsinki with approval from the Ethics Committee of Beijing Children's Hospital. Written informed consent was obtained from all the parents of each child as well as their own participation in the study.

\section{TCIRG1 gene mutation analysis}

Blood samples were collected from patients (families) with IMO at Beijing Children's Hospital, Capital Medical University. Twenty-seven patients with TCIRG1-related osteopetrosis were included in the study by gene detection. We performed mutation analysis of the TCIRG1 gene using direct DNA sequencing of PCR-amplified exons (the mutation nomenclature conforms to www. hgvs.org/mutnomen). The TCIRG1 gene was amplified from genomic DNA (genomic sequence AF033033) in the conditions previously described. ${ }^{6}$ Prediction of the protein domains was based on the potential topological domains reported in UniProt database (Q13488; www. uniprot.org) and refined by homology with the yeast subunit a (Vph1p). ${ }^{9}$ The putative structural consequences of mutations were estimated in silico by sequence analysis and multiple sequence alignment information. Patients with other gene-related osteopetrosis were excluded. None of these Chinese probands were the offspring of a consanguineous marriage. Of the 27 patients with IMO, 12 had a frameshift mutation (the frameshift mutation group, group F) while 15 had a point mutation (the point mutation group, group $\mathrm{P}$ ).

\section{Clinical examination}

A clinical diagnosis of osteopetrosis was made on the basis of the following criteria at presentation (which has been described by the previous study ${ }^{10}{ }^{11}$ : early symptom onset (before 2 years of age), high bone mineral density, bone marrow failure with consequent pancytopenia, hepatosplenomegaly and cranial nerve palsies by the haematology specialists. The ophthalmological features at the onset of IMO were ascertained in all 27 cases by examining the clinical records at Beijing Children's Hospital. We compared the group $\mathrm{F}$ with the group $\mathrm{P}$ in terms of sex, age at diagnosis of IMO and appearance of eye (eg, gaze properties including nystagmus, strabismus, optic atrophy, optic canal diameter and waveform of Flash visual-evoked potential (FVEP)). For analysis, the onset of IMO was defined as the time of the initial diagnosis.

We checked the gaze qualities with a light source, identifying whether there was nystagmus and/or strabismus. Slit lamp and dilated Retcam III fundus examination were performed after applying tropicamide eye-drops.

Data for orbital 3D-CT reconstruction were collected for all children using the Discovery 750 (GE Healthcare) with bone window parameters and layer thickness less than $2 \mathrm{~mm}$. The optic canal diameter was measured independently by two radiologists and reviewed by the third radiologist. Children were in the supine position with a cushion under their heads. Axial scanning was used, and the reconstruction was multiplanar reconstruction. The scanning parameters were as follows: tube volt $80 \mathrm{kV}$; pitch 1.375; depth $1 \mathrm{~mm}$; interlamellar spacing $1 \mathrm{~mm}$; and a $256 \times 256$ matrix. The average radiation dose in this study was $1.09 \pm 0.02 \mathrm{mGy}$, which is much lower than the American College of Radiology CT accredited CT Dosimetry Index reference level of $75 \mathrm{mGy}$. A low-dose CT protocol for children was followed for the purpose of this study.

FVEP was examined using a visual electrophysiology system (Roland, Germany). The standard FVEP protocol recommended by the International Standard EEG 10-20 System (International Society for Clinical Electrophysiology of Vision) was followed: the examination was performed in a darkroom; the electrodes including the active electrode, reference electrode and ground electrode were placed; the eyes were stimulated by a mini handheld stimulator separately and repeated twice per eye, with more than 70 superposition times. The excitation frequency was set at $30 \mathrm{~Hz}$.

\section{Statistical analysis}

Statistical analyses were performed using SPSS V.13.0. In two groups, gaze qualities, optic atrophy and FVEP waveform were respectively compared by Pearson $\chi^{2}$, age at onset by Mann-Whitney $U$ test and optic canal diameter by Student's t-test. Alpha correct $\mathrm{p}$ values for five comparisons $(0.05 / 5=0.01)$ were considered as statistically significant.

\section{RESULTS}

All cases in group $\mathrm{F}$ and group $\mathrm{P}$ had ocular symptoms and signs of IMO such as loss of vision, optic canal stenosis, with/without nystagmus, strabismus, or optic atrophy. The clinical and genetic data of the 27 patients with TCIRG1 mutations were summarised in table 1.

The male:female ratio was 7:5 in the frameshift mutation group and 9:6 in the point mutation group. The mean age at onset was 1.8 months in group $\mathrm{F}$ and 4.3 months in group $\mathrm{P}$, but there was no significant difference in these two groups (table 2; Mann-Whitney $\mathrm{U}$ test, $\mathrm{p}=0.028<0.05)$.

Significant differences in the clinical findings were found between the two groups (table 2). Patients with poor gaze qualities, which referred to no response to moving object/light, nystagmus or strabismus, included $22(92 \%)$ of 24 eyes with frameshift mutation and 16 $(53 \%)$ of 30 eyes with point mutation (Pearson $\chi^{2}$; $\mathrm{p}=0.002<0.01)$. We also found that there were 16 eyes $(67 \%)$ with optic atrophy of 24 eyes in the frameshift mutation cases, and $6(20 \%)$ of 30 eyes in the point mutation cases (Pearson $\chi^{2} ; \mathrm{p}=0.001<0.01$ ). Orbital 3D-CT showed that the average optic canal diameter was 1.45 $\mathrm{mm}$ in the 12 frameshift mutation cases, $1.87 \mathrm{~mm}$ in the 


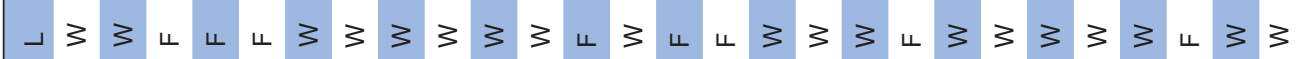

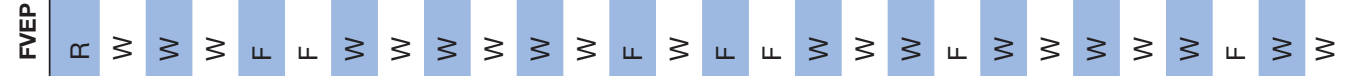

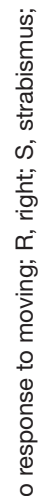

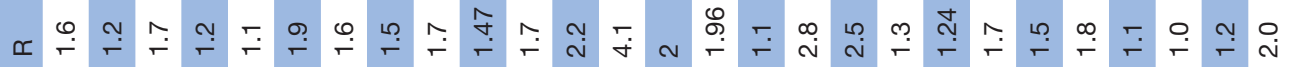

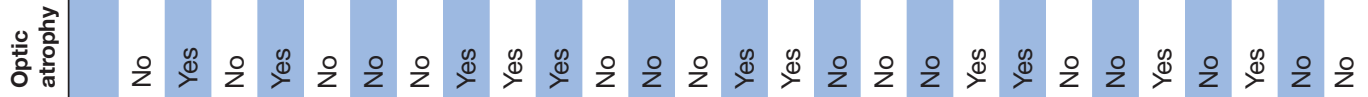

$\sum_{\substack{\infty \\ 2}}^{\infty}$

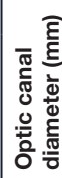

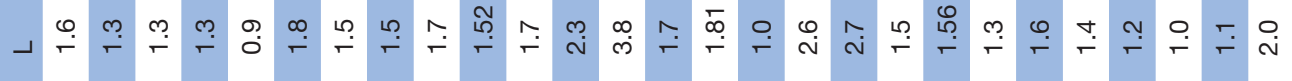

일

픙

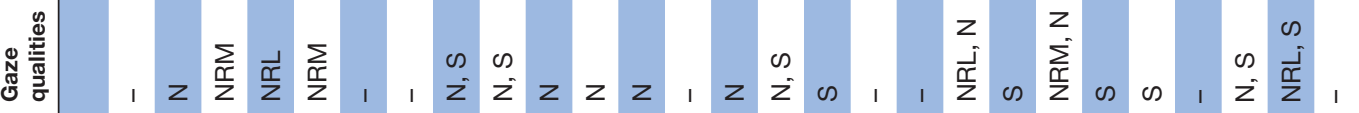

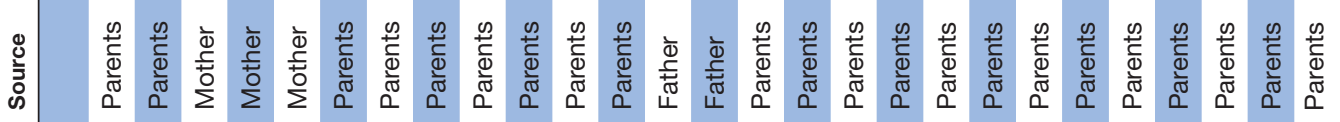




\begin{tabular}{|c|c|c|c|c|c|}
\hline Group (cases/yes) & $\begin{array}{l}\text { Mean age at onset } \\
\text { (month) }\end{array}$ & Poor gaze qualities $†$ & Optic atrophy† & $\begin{array}{l}\text { Optic canal diameter } \\
(\mathrm{mm}) \ddagger\end{array}$ & Flat FVEP† \\
\hline Group F (12/24) & $1.8(0-6)$ & 22 & 16 & $1.45 \pm 0.36$ & 10 \\
\hline Group P (15/30) & $4.3(0-17)$ & 16 & 6 & $1.87 \pm 0.73$ & 5 \\
\hline$P$ values & 0.028 & 0.002 & 0.001 & 0.009 & 0.042 \\
\hline
\end{tabular}

*Mann-Whitney $U$ test; $p<0.01$.

†Pearson $\chi^{2} ; p<0.01$.

¥Student's t-test; $p<0.01$.

FVEP, Flash visual-evoked potential; IMO, infantile malignant osteopetrosis; TCIRG1, T-cell immune regulator 1.

15 point mutation cases (Student's t-test; $\mathrm{p}=0.009<0.01$ ). FVEP indicated that the waveforms in 10 eyes $(42 \%)$ were not elicited in group $\mathrm{F}$, yet five eyes $(17 \%)$ in group $\mathrm{P}$ (Pearson $\chi^{2} ; \mathrm{p}=0.042>0.01$ ).

The clinical features of one case (case 18) with point mutation and two siblings (cases 25 and 26) with frameshift mutation were presented in this study.

\section{Case 18}

A female Chinese infant who had bilateral hip asymmetry and anaemia at 6 months of age was referred to the Department of Hematology, Beijing Children's Hospital, and diagnosed with IMO with general examination. No contributory family history or consanguinity was present. Genetic analysis showed that she was a compound heterozygote with both c. $117+5 \mathrm{G}>\mathrm{A}$ (from the father) and c.630G $>\mathrm{A}$ (from the mother) mutations. Both eyes had a good fixation ability without nystagmus or strabismus. The diameter of optic canals was $2.5 \mathrm{~mm}$ in the right eye and $2.7 \mathrm{~mm}$ in the left eye. Optic atrophy was found in neither eye by Retcam III fundus examination. The latency (P2) of the FVEP was $117.1 / 123.5 \mathrm{~ms}$ and the amplitude was 33.2/32.7 $\mu \mathrm{V}$ in the right and left eyes, respectively, which indicated relatively good conductivity of the optic nerve. After 8 months, she received successful haematopoietic stem cell transplantation (HSCT), and the optic canal stenosis was controlled for 10 months. The latest FVEP results manifested no reduction in conductivity of the optic nerve, and the Teller visual acuity for both eyes was 20/63.

\section{Case 25 and case 26 (brother and sister)}

These two siblings were homozygotes for c.1371delC. Their parents were no consanguineous couple.

The brother was referred to the Beijing Children's Hospital as newborn for developmental retardation and strabismus. Various potentially fatal manifestations had been ascertained by haematologists, such as bone marrow failure resulting in pancytopenia, hepatosplenomegaly, growth retardation and cranial nerve palsies. He was affected by bilateral proptosis, nystagmus and alternating exotropia, with an extremely poor fixation ability and optic atrophy. The diameter of optic canals was 1.0 $\mathrm{mm}$ in both eyes, and showed severe compression of optic canals. The waveform of FVEP was not elicited. The child had died from pulmonary infection and multiple organ failure before he waited for the right donor for HSCT.
After 3 years, the boy's sister was born, whose clinical features were very similar to her brother. Within 1 week of age, the neonate was also referred to the Beijing Children's Hospital and diagnosed with IMO by the haematologist. Her eyes were characterised by no response to light and alternating exotropia. Optic nerve atrophy has not yet been shown in the fundus examination. However, the compression of optic canals has been very obvious, and its diameter was 1.2 and $1.1 \mathrm{~mm}$, respectively. P2 latency period of FVEP was $171 / 183 \mathrm{~ms}$ and the amplitude was $1.92 / 1.86 \mu \mathrm{V}$ in bilateral eyes, which revealed severe conduction block of the optic nerve. AlloHSCT was performed at 5 months of age; unfortunately, as a result of acute respiratory distress syndrome, she died 28 days after bone marrow transplant.

\section{DISCUSSION}

Osteopetrosis is a heterogeneous disease, depending on the causal genes, modes of inheritance and disease severity (phenotypes). To our knowledge, this is the first study on the ophthalmic phenotypes of TCIRG1 mutations in Chinese patients with IMO. Our result showed that 12 cases had a frameshift mutation and 15 cases had a point mutation in the TCIRG1 gene.

First, from the aspect of onset, $58 \%(7 / 12)$ cases in the frameshift mutation group and $20 \%(3 / 15)$ cases in the point mutation group were severely affected in neonate, even in fetus. We found that, clinically, the cases in the TCIRG1 frameshift mutation group had a little earlier IMO onset age, compared with those from point mutation group; however, there were no statistical differences between the two groups. This may partly be explained by selection bias. Theoretically, the children from frameshift mutation group may have more severe phenotype, thus could be more easily attended and identified. On the other hand, children from point mutation group may have more latent onset or slight symptom, thus could be more easily ignored. For example, three patients (cases 4, 19, 23) who were treated with poor vision as the first symptom all had frameshift mutation in the TCIRG1 gene.

Second, from the aspect of severity, most of the TCIRG1-related patients $(88.9 \%, 24 / 27)$ showed a severe ophthalmic phenotype characterised by optic canal stenosis with or without optic atrophy, nystagmus and strabismus. Moreover, the above ocular features from 
frameshift mutation cases were all more serious than those from point mutation cases. This finding can be ascribed to the fact that most TCIRG1 mutations, being frameshifts or stop codons, completely disrupt the protein function. ${ }^{12}$ For example, the two siblings (cases 25 and 26) who were homozygotes for c.1371delC had typical frameshift mutation, changing the reading frame (the grouping of the codons) and resulting in a completely different translation from the original. There was a similarity in the phenotypic features due to the same genetic factor in these two siblings, suggesting the decisive role of the TCIRG1 mutation of the disease.

Interestingly, we also found $18.5 \%(5 / 27)$ of cases with no frameshift mutation but suffered from severely impaired vision. For these three cases, the point mutations were all inherited from either of their parents who only carried one mutation and behaved healthy. We hypothesise that the following facts may explain such inconsistency: IMO is a sort of recessive disease, yet in some cases, only one allele was detected, or, as shown in table 1, the mutant gene came from only one parent. This means the mutation could have been missed in the sequencing, or there is a large deletion. It is also possible that there are two heterozygous genes at different sites that have not been detected. Kornak et al, Sobacchi et al and Susani et al had ever reported this phenomenon. ${ }^{6} 1213$ The best explanation is that maybe an additional undetected mutation exists in these cases. On the other hand, not all of them had typical phenotypic features. $11.1 \%$ $(3 / 27)$ of cases had relatively wider optic canal diameter, better optic nerve conduction in FVEP and more stable fixation ability at the age of onset. We did not know whether some environmental factors might modulate the severity of the disease, in addition to the potential for genetic modifiers. Anyway, such extraordinary cases showed that the clinical features of IMO were the result of a comprehensive effect.

To evaluate the clinical features, VEP supposed to be more valuable. It provides early clues for visual pathway disorders, and the electrophysiological changes are more sensitive than radiological abnormalities. However, for some cases, CT scan showed the extent of canal stenosis was significantly different between frameshift and point mutation groups, and VEP showed no difference in function. The answer is yet to know.

This study had some limitations. The small number of cases and the abnormal distributed data on onset age influenced the accuracy of statistical tests. It is expected to continue to observe the differences in FVEP data by increasing the sample size. In the future, the mutation at different loci in different genotypes should be assayed in both parents.

In conclusion, among Chinese patients with TCIRG1-related IMO, we found that the phenotypes of eyes were more severe in cases with frameshift mutations. Illuminating how the TCIRG1 gene mutation is related to clinical characteristics may encourage better understanding of the mechanism of ocular sign occurrence and more targeted treatment of IMO.

Acknowledgements Haematologists Maoquan Qin and Guanghua Zhu are acknowledged for providing the genetic data of the patients.

Contributors WC carried out CT and FVEP study, performed the statistical analysis and drafted the manuscript. QW participated in the design of the study and performed the statistical analysis. WW conceived the study and participated in its design and coordination and helped draft the manuscript. All authors read and approved the final manuscript.

Funding This study was supported by grants from the National Natural Science Foundation of China (81570891); the National Natural Science Foundation of China (81272981); the Beijing Municipal Administration of Hospitals' Ascent Plan (DFL20150201); the Science and Technology Project of Beijing Municipal Science and Technology Commission (Z151100001615052); the Beijing Municipal Administration of Hospitals Clinical Medicine Development of Special Funding Support (ZYLX201307); the Beijing Natural Science Foundation (7151003); and the Advanced Health Care Professionals Development Project of Beijing Municipal Health Bureau (2014-2-003).

Competing interests None declared.

Patient consent Parental/guardian consent obtained.

Ethics approval Ethics Committee of Beijing Children's Hospital

Provenance and peer review Not commissioned; internally peer reviewed.

Open access This is an open access article distributed in accordance with the Creative Commons Attribution Non Commercial (CC BY-NC 4.0) license, which permits others to distribute, remix, adapt, build upon this work non-commercially, and license their derivative works on different terms, provided the original work is properly cited, appropriate credit is given, any changes made indicated, and the use is non-commercial. See: http://creativecommons.org/licenses/by-nc/4.0

\section{REFERENCES}

1. Warman ML, Cormier-Daire V, Hall C, et al. Nosology and classification of genetic skeletal disorders: 2010 revision. Am J Med Genet A 2011;155A:943-68.

2. Sobacchi C, Schulz A, Coxon FP, et al. Osteopetrosis: genetics, treatment and new insights into osteoclast function. Nat Rev Endocrinol 2013;9:522-36.

3. Stark Z, Savarirayan R. Osteopetrosis. Orphanet J Rare Dis 2009;4:5.

4. Frattini A, Orchard PJ, Sobacchi C, et al. Defects in TCIRG1 subunit of the vacuolar proton pump are responsible for a subset of human autosomal recessive osteopetrosis. Nat Genet 2000;25:343-6.

5. Teitelbaum SL, Ross FP. Genetic regulation of osteoclast development and function. Nat Rev Genet 2003;4:638-49.

6. Sobacchi C, Frattini A, Orchard P, et al. The mutational spectrum of human malignant autosomal recessive osteopetrosis. Hum $\mathrm{Mol}$ Genet 2001;10:1767-73.

7. Pangrazio A, Caldana ME, Lo lacono N, et al. Autosomal recessive osteopetrosis: report of 41 novel mutations in the TCIRG1 gene and diagnostic implications. Osteoporos Int 2012;23:2713-8.

8. Palagano E, Susani L, Menale C, et al. Synonymous mutations add a layer of complexity in the diagnosis of human osteopetrosis. J Bone Miner Res 2017;32:99-105.

9. Wang Y, Toei M, Forgac M. Analysis of the membrane topology of transmembrane segments in the C-terminal hydrophobic domain of the yeast vacuolar ATPase subunit a (Vph1p) by chemical modification. J Biol Chem 2008;283:20696-702.

10. Frattini A, Pangrazio A, Susani L, et al. Chloride channel CICN7 mutations are responsible for severe recessive, dominant, and intermediate osteopetrosis. J Bone Miner Res 2003;18:1740-7.

11. Del Fattore A, Cappariello A, Teti A. Genetics, pathogenesis and complications of osteopetrosis. Bone 2008;42:19-29.

12. Susani L, Pangrazio A, Sobacchi C, et al. TCIRG1-dependent recessive osteopetrosis: mutation analysis, functional identification of the splicing defects, and in vitro rescue by U1 snRNA. Hum Mutat 2004;24:225-35.

13. Kornak U, Schulz A, Friedrich $W$, et al. Mutations in the a3 subunit of the vacuolar $\mathrm{H}(+)$-ATPase cause infantile malignant osteopetrosis. Hum Mol Genet 2000;9:2059-63. 